\title{
INVESTIGATION OF HEALING EFFECTS OF THERMAL SPRING WATER AND MUD ON EXPERIMENTALLY- INDUCED ATOPIC DERMATITIS IN MICE
}

\author{
Bulent Elitok $^{1 *}$, Sinem Odabasi ${ }^{1}$
}

${ }^{1}$ Department of Internal Medicine, Faculty of Veterinary Medicine, Afyon Kocatepe University

Afyonkarahisar/Turkey

*Corresponding author:

Assoc. prof. dr. Bulent Elitok

Department of Internal Medicine

Faculty of Veterinary Medicine, Afyon

Kocatepe University,

Afyonkarahisar, Turkey

Phone:+902724440303

ORCID ID: 0000-0003-3336-4479

E-mail: elitok1969@hotmail.com

Original submission:

20 April 2020

Revised submission:

27 May 2020

Accepted:

06 June 2020

\section{ABSTRACT}

This study was conducted to determine the therapeutic efficacy of hot spring water and mud in atopic dermatitis. For this purpose, totally 40 Albino mice of the same daily age were used. All mice were treated with 2\% 2,4-dinitrochlorobenzene after de-epilation in the dorsal region for creating atopic dermatitis. After disease formation, a 21-day treatment period was begun, and the mice were equally and randomly divided into two groups, control and study. Inter and intra-group comparisons of clinical, hematological and biochemical blood findings measured before and after the formation of atopic dermatitis, and on days $1,7,14$ and 21 of the treatment period were performed in this study. In comparison with the control group on the last day of the study, it was seen that the body weight gain along with total leukocyte, neutrophil, monocyte, eosinophil counts and AST, LDH, GGT, OCT and IgE levels were significantly $(\mathrm{p}<0.05)$ decreased in the study group, whereas the mean heart and respiratory frequencies, erythrocyte and lymphocyte counts along with hemoglobin and hematocrit levels remarkably increased $(p<0.05)$. There was no significant differences between the groups in terms of basophil count. Last day tissue samples showed that skin damage was significantly improved in the study group of animals, while it continued in the control group mice. Consequently, it was concluded that atopic dermatitis was improved in mice treated with Süreyya I hot spring water along with mud.

Keywords: Afyonkarahisar, atopic dermatitis, balneotherapy, mouse, treatment 


\section{INTRODUCTION}

Atopic dermatitis (AD) is a constantly recurring and chronic skin disease that develops as a result of Th2 dominant hyperimmune disorders, and its incidence is increasing especially in industrialized countries (Boguniewicz and Leung, 2010). It has a complex pathogenesis, including environment that consists of substances that irritate the skin, impaired skin barrier function, and influences of immunological factors (Hwang et al., 2013). Symptoms such as itching, dandruff, dryness and inflammation are the most common symptoms that occur during the disease (Boguniewicz and Leung, 2010).

It is well known that spa products have been used in dermatological diseases for a long time with various methods (Matz et al., 2003). In addition to being beneficial for rheumatoid diseases, it has been reported in the studies that spa muds are also widely used in the treatment of diseases such as acne, roseola, psoriasis, and the results that have been achieved (Cozzi et al., 1995). It is also reported that balneotherapy applications have very beneficial results in dermatitis cases (Elitok, 2011).

The aim of this study was to determine the effectiveness of Süreyya I Thermal Spring water and mud in the treatment of atopic dermatitis at first.

\section{MATERIALS AND METHODS}

The experimental part of this study was implemented in Afyon Kocatepe University Experimental Animals Application and Research Center, in accordance with the Directive of Afyon Kocatepe University Experimental Animals
Ethics Committee (AKUHADYEK) with the report numbered 60-18, and supported by Afyon Kocatepe University Scientific Research Projects (AKÜBAPK) and with the report as Master's Thesis Project with 18. SAĞ.BİL.14 reference number.

\section{Animals}

Totally 40 Albino mice of 8 weeks of age were used in this study. All animals were kept in the cages for 12 hours during night and 12 hours during day under the stable conditions, same humidity and temperature. All the mice were fed with mouse food ad libitum.

\section{Creating Atopic Dermatitis}

Before applying the atopic dermatitis procedure, 4 out of 40 mice were exempted to collect blood samples. Then, 2-4-dinitrochlorobenzene (DNCB) (Sigma Aldrich, St Louis, MO, USA) solved in acetone/olive oil solution ( $2 \%$ acetone in olive oil) was applied to the shaved area. After 3 days of this application, $150 \mu \mathrm{g}$ of mite extract (Dermatophagoides farinae) was dissolved in PBS containing $0.5 \%$ Tween 20 and applied to the same area on the back, and AD was created (Hwang et al., 2013).

\section{Groups and Treatment Procedure}

After the formation of AD, 4 out of 36 remaining mice were ex-exhausted for blood and histopathological examinations. The remaining 32 mice with $\mathrm{AD}$ were divided equally and randomly into 2 groups for 21 days of treatment period as below:

1. Control Group (CG): 16 mice with experimental AD served as CG. CG mice were fed fresh tap water daily, and their feed and water were $a d$ 
libitum. All the mice in this group were also bathed in tap water with a temperature of $35 \pm 2{ }^{\circ} \mathrm{C}$ for 15 minutes every day.

2. Study Group (SG): The remaining 16 mice with $\mathrm{AD}$ were assigned to $\mathrm{SG}$. The mice in this group were fed with Süreyya I hot spring fresh water ad libitum. All the mice in this group were alse bathed in Süreyya I hot spring water every day with a temperature of $35 \pm 2^{\circ} \mathrm{C}$ for 15 minutes. Additionally, all the $\mathrm{SG}$ mice received hot mud obtained from Süreyya I Spa Source, accumulating from running water. The mud was applied to the area of experimentally created lesions for 30 minutes, before bathing twice a week.

Süreyya I Hot Spring Water, used for the purpose of treatment in this study, has a total mineral content of $4046.8 \mathrm{~g} / \mathrm{L}$ and belongs to the thermomineral water group with high sodium bicarbonate, carbon dioxide, magnesiun, calcium, fluoride and silicon (Çalışkan, 2019).

\section{Methods}

Body temperatures $(\mathrm{T})$, respiratory rates $(\mathrm{R})$ and heart frequencies $(\mathrm{P})$ of all mice used in the study were measured before the study, after AD formation, and on the 1st, 7th, 14th and 21 st day of treatment.

\section{Hematological Examinations}

Hematologically, erythrocyte (RBC), total leukocyte (WBC), hematocrit (HCT), hemoglobin (HB), mean corpuscular volume (MCV), mean corpuscular hemoglobin (MHC), mean corpuscular hemoglobin concentration (MCHC), lymphocyte (L), leukogram such as neutrophils $(\mathrm{N})$, eosinophils (E), monocytes (M) and basophils (B) were counted by Chemray Brand blood count device using commercial test kits.

\section{Blood Biochemical Examinations}

In the blood biochemical examinations, serum aspartate aminotransferase (AST), gammaglutamyl transferase (GGT), lactate dehydrogenase (LDH), ornityl-carbomyl transferase (OCT), total protein (TP), albumin (ALB) and glucose (GLU) were measured using Cobas Integra 400 Plus Roche The brand (Roche Diagnostics GmbH, Germany) by the analyzer. Immunoglobulin E (IgE) levels were measured by ChemWell Elisa Reader (ChemWell Chromate 4300 Elisa Reader, Awareness Technology, Inc. Martin Hwy. Palm City, USA) Elisa kits (Sunred Biological Technology Company Co. Shangai/China).

\section{Histopathological Examinations}

After the formation of atopic dermatitis and on the 1st, 7th, 14th and 21st days of treatment, the skin samples of 4 randomly selected mice from $\mathrm{CG}$ and SG were taken under anesthesia $(100 \mathrm{mg} / \mathrm{kg}$ of ketamine, $10 \mathrm{~m} / \mathrm{kg}$ xylazine) (Suckow et al., 2012). These samples were sent to Veterinary Control Central Research Institute Pathology Laboratory to perform histopathological examinations in $10 \%$ formol, where 5 micron thick sections were taken, stained with hematoxylin-eosin and examined in a light microscope.

\section{Statistical Analyses}

Statistical calculations for CG and SG animals were made in accordance with the variance analysis (ANOVA) method. The Duncan test was used to determine the significance of the differencein the study group. Statistical analyzes were provided through the Windows-compatible SPSS 18.1 (Inc., Chicago, II, USA) package program. The data levels obtained are presented as mean \pm standard error and $\mathrm{p}<0.05$ is considered to be significant. 


\section{RESULTS}

Since the production of animals started at the same time ,and the animals were about eight weeks old, there was no age difference between the animals ( $>$ $>0.05$ ). Body weights (bw) of the animals were electronically measured individually, and on weighing performed on the 21 st day after the treatment, observed were bw average of $29.0 \mathrm{~g}$ for $\mathrm{CG}$ and $28.3 \mathrm{~g}$ for SG. It was observed that a statistically significant difference $(p<0.05)$ occurred in terms of their bw mean.

\section{Clinical Findings}

There was no significant difference in terms of $\mathrm{T}$ mean when compared to time periods and the mean of $\mathrm{CG}$ and $\mathrm{SG}$ after the formation of $\mathrm{AD}(\mathrm{p}>0.05)$. It was determined that significant differences ( $p$ $<0.05$ ) were formed in terms of $\mathrm{P}$ and $\mathrm{R}$, in the inter-group and intra-group comparisons, the most important increases seen in SG animals when the difference was statistically significant $(\mathrm{p}<0.05)$.

\section{Hematological Findings}

Hematological examination findings are shown in Table 1.

Table 1 Hematological findings of the animals

\begin{tabular}{|c|c|c|c|c|c|c|c|c|}
\hline \multicolumn{2}{|c|}{$\begin{array}{c}\text { Measurement } \\
\text { Time/Parameters }\end{array}$} & \multirow{2}{*}{$\begin{array}{c}\text { WBC } \\
\left(10^{3} / \mathbf{m m 3}\right)\end{array}$} & \multirow{2}{*}{$\begin{array}{c}\text { RBC } \\
\left(10^{6} / \mathrm{mm} 3\right)\end{array}$} & \multirow{2}{*}{$\begin{array}{c}\text { HB } \\
(\mathrm{g} / \mathrm{dl})\end{array}$} & \multirow{2}{*}{$\begin{array}{r}\text { HCT } \\
(\%) \\
X \pm \text { SD }\end{array}$} & \multirow{2}{*}{$\begin{array}{c}\text { MCV } \\
\text { (fl) } \\
\mathrm{X} \pm \text { SD }\end{array}$} & \multirow{2}{*}{$\begin{array}{c}\text { MCH } \\
\text { (pg) }\end{array}$} & \multirow{2}{*}{$\begin{array}{c}\text { MCHC } \\
\text { (g/dl) }\end{array}$} \\
\hline & Groups & & & & & & & \\
\hline $\begin{array}{c}\text { BS } \\
(\mathrm{n}=40)\end{array}$ & - & $8.42 \pm 2.30^{\mathrm{c}}$ & $\begin{array}{l}8.02 \pm \\
1.24^{\mathrm{ab}}\end{array}$ & $\begin{array}{l}14.01 \pm \\
2.37^{\mathrm{a}}\end{array}$ & $\begin{array}{l}44.11 \pm \\
4.12^{\mathrm{b}}\end{array}$ & $\begin{array}{l}54.86 \pm \\
2.14^{\mathrm{c}}\end{array}$ & $\begin{array}{l}17.47 \pm \\
2.34^{\mathrm{a}} \\
\end{array}$ & $\begin{array}{l}31.82 \pm \\
3.04^{\mathrm{a}}\end{array}$ \\
\hline $\begin{array}{l}\text { AADF } \\
(\mathrm{n}=36)\end{array}$ & - & $\begin{array}{l}11.32 \pm \\
2.30^{\mathrm{ab}} \\
\end{array}$ & $\begin{array}{l}7.64 \pm \\
1.22^{\mathrm{b}} \\
\end{array}$ & \begin{tabular}{|l|}
$13.68 \pm$ \\
$2.16^{\mathrm{b}}$ \\
\end{tabular} & $\begin{array}{l}44.78 \pm \\
4.12^{b}\end{array}$ & $\begin{array}{l}58.12 \pm \\
3.12^{\mathrm{a}} \\
\end{array}$ & $\begin{array}{l}17.68 \pm \\
3.43^{\mathrm{a}} \\
\end{array}$ & $\begin{array}{l}30.67 \pm \\
3.17^{\mathrm{b}} \\
\end{array}$ \\
\hline \multirow{2}{*}{$\begin{array}{c}\text { AT } \\
\text { 1st Day }\end{array}$} & $\begin{array}{l}\mathrm{CG} \\
(\mathrm{n}=16)\end{array}$ & $\begin{array}{l}12.04 \pm \\
2.20^{\mathrm{a}}\end{array}$ & $\begin{array}{l}7.76 \pm \\
0.68^{\mathrm{b}}\end{array}$ & $\begin{array}{l}13.56 \pm \\
1.47^{\mathrm{b}} \\
\end{array}$ & $\begin{array}{l}45.01 \pm \\
4.24^{\mathrm{ab}}\end{array}$ & $\begin{array}{l}57.43 \pm \\
3.10^{\mathrm{b}} \\
\end{array}$ & $\begin{array}{l}17.26 \pm \\
3.23^{\mathrm{a}} \\
\end{array}$ & $\begin{array}{l}30.16 \pm \\
1.68^{\mathrm{b}} \\
\end{array}$ \\
\hline & $\begin{array}{l}\mathrm{S} \\
(\mathrm{n}=16)\end{array}$ & $\begin{array}{l}11.37 \pm \\
2.05^{\mathrm{ab}} \\
\end{array}$ & $\begin{array}{l}8.03 \pm \\
0.58^{\mathrm{ab}}\end{array}$ & \begin{tabular}{|l|}
$14.03 \pm$ \\
$1.36^{\mathrm{a}}$ \\
\end{tabular} & $\begin{array}{l}44.58 \pm \\
3.25^{\mathrm{b}}\end{array}$ & $\begin{array}{l}55.00 \pm \\
1.65^{\mathrm{c}} \\
\end{array}$ & \begin{tabular}{|l|}
$17.48 \pm$ \\
$3.08^{\mathrm{a}}$ \\
\end{tabular} & $\begin{array}{l}31.51 \pm \\
1.57^{\mathrm{a}} \\
\end{array}$ \\
\hline \multirow{2}{*}{$\begin{array}{l}\text { AT } \\
\text { 7th } \\
\text { Day }\end{array}$} & $\begin{array}{l}\mathrm{CG} \\
(\mathrm{n}=12)\end{array}$ & $\begin{array}{l}12.00 \pm \\
1.28^{\mathrm{a}}\end{array}$ & $\begin{array}{l}8.00 \pm \\
0.50^{\mathrm{ab}}\end{array}$ & $\begin{array}{l}13.72 \pm \\
1.10^{\mathrm{b}} \\
\end{array}$ & $\begin{array}{l}45.34 \pm \\
2.67^{\mathrm{a}}\end{array}$ & $\begin{array}{l}56.71 \pm \\
1.37^{\mathrm{a}}\end{array}$ & $\begin{array}{l}17.12 \pm \\
1.48^{\mathrm{a}} \\
\end{array}$ & $\begin{array}{l}30.52 \pm \\
0.67^{\mathrm{b}}\end{array}$ \\
\hline & $\begin{array}{l}S \\
(n=12)\end{array}$ & $\begin{array}{l}11.03 \pm \\
1.22^{\mathrm{ab}}\end{array}$ & $\begin{array}{l}8.10 \pm \\
0.44^{\mathrm{ab}}\end{array}$ & $\begin{array}{l}14.11 \pm \\
1.00^{\mathrm{a}} \\
\end{array}$ & $\begin{array}{l}45.10 \pm \\
2.44^{\mathrm{ab}}\end{array}$ & $\begin{array}{l}55.53 \pm \\
1.26^{\mathrm{c}} \\
\end{array}$ & $\begin{array}{l}17.40 \pm \\
1.37^{\mathrm{a}} \\
\end{array}$ & $\begin{array}{l}31.29 \pm \\
0.55^{\text {ab }}\end{array}$ \\
\hline \multirow{2}{*}{$\begin{array}{l}\text { AT } \\
\text { 14th } \\
\text { Day }\end{array}$} & $\mathrm{CG}(\mathrm{n}=8)$ & \begin{tabular}{|l|}
$11.82 \pm$ \\
$0.67^{\mathrm{ab}}$ \\
\end{tabular} & $\begin{array}{l}7.89 \pm \\
0.37^{\mathrm{b}} \\
\end{array}$ & \begin{tabular}{|l}
$14.01 \pm$ \\
$0.62^{\mathrm{a}}$ \\
\end{tabular} & $\begin{array}{l}44.88 \pm \\
1.27^{\mathrm{b}} \\
\end{array}$ & $\begin{array}{l}54.64 \pm \\
0.57^{\mathrm{c}} \\
\end{array}$ & \begin{tabular}{|l}
$17.54 \pm$ \\
$0.62^{\mathrm{a}}$ \\
\end{tabular} & $\begin{array}{l}31.22 \pm \\
0.38^{\mathrm{a}} \\
\end{array}$ \\
\hline & $\mathrm{SG}(\mathrm{n}=8)$ & $9.43 \pm 0.60^{b}$ & $\begin{array}{l}8.23 \pm \\
0.24^{\mathrm{a}} \\
\end{array}$ & \begin{tabular}{|l}
$14.18 \pm$ \\
$0.57^{\mathrm{a}}$ \\
\end{tabular} & $\begin{array}{l}45.14 \pm \\
1.32^{\mathrm{ab}} \\
\end{array}$ & $\begin{array}{l}54.92 \pm \\
0.36^{\mathrm{c}} \\
\end{array}$ & $\begin{array}{l}17.22 \pm \\
0.47^{\mathrm{a}} \\
\end{array}$ & $\begin{array}{l}31.41 \pm \\
0.36^{\mathrm{a}} \\
\end{array}$ \\
\hline \multirow{2}{*}{$\begin{array}{c}\text { AT } \\
\text { 21th } \\
\text { Day }\end{array}$} & $\mathrm{CG}(\mathrm{n}=4)$ & $\begin{array}{l}11.05 \pm \\
0.34^{\mathrm{ab}}\end{array}$ & $\begin{array}{l}8.01 \pm \\
0.17^{\mathrm{ab}}\end{array}$ & $\begin{array}{l}13.82 \pm \\
0.31^{\mathrm{b}}\end{array}$ & $\begin{array}{l}45.56 \pm \\
0.54^{\mathrm{a}}\end{array}$ & $\begin{array}{l}56.55 \pm \\
0.24^{\mathrm{ab}}\end{array}$ & $\begin{array}{l}17.18 \pm \\
0.38^{\mathrm{a}} \\
\end{array}$ & $\begin{array}{l}30.33 \pm \\
0.17^{\mathrm{b}}\end{array}$ \\
\hline & $\mathrm{SG}(\mathrm{n}=4)$ & $8.68 \pm 0.36^{c}$ & $\begin{array}{l}8.41 \pm \\
0.15^{\mathrm{a}}\end{array}$ & $\begin{array}{l}14.07 \pm \\
0.16^{\mathrm{a}}\end{array}$ & $\begin{array}{l}45.89 \pm \\
0.62^{\mathrm{a}}\end{array}$ & $\begin{array}{l}54.72 \pm \\
0.21^{\mathrm{c}}\end{array}$ & $\begin{array}{l}16.70 \pm \\
0.35^{\mathrm{b}}\end{array}$ & $\begin{array}{l}30.65 \pm \\
0.16^{\mathrm{b}}\end{array}$ \\
\hline
\end{tabular}




\section{Continuation of Table 1}

\begin{tabular}{|c|c|c|c|c|c|c|c|c|}
\hline \multicolumn{2}{|c|}{$\begin{array}{c}\text { Measurement } \\
\text { Time/Parameters }\end{array}$} & \multirow{2}{*}{$\begin{array}{c}\text { MCH } \\
(\mathbf{p g})\end{array}$} & \multirow{2}{*}{$\begin{array}{c}\text { MCHC } \\
(\mathrm{g} / \mathrm{dl}) \\
\mathbf{X} \pm \mathbf{S D}\end{array}$} & $\begin{array}{r}\mathbf{L} \\
\%\end{array}$ & $\begin{array}{l}\mathbf{N} \\
\%\end{array}$ & $\begin{array}{r}\mathbf{E} \\
\%\end{array}$ & \multirow{2}{*}{$\begin{array}{c}\begin{array}{c}\mathbf{M} \\
\%\end{array} \\
\mathbf{X} \pm \mathbf{S D}\end{array}$} & \multirow{2}{*}{$\begin{array}{c}\text { B } \\
\% \\
X \pm S D\end{array}$} \\
\hline & Groups & & & $\mathbf{X} \pm \mathrm{SD}$ & $\mathbf{X} \pm \mathbf{S D}$ & $\mathbf{X} \pm \mathbf{S D}$ & & \\
\hline $\begin{array}{c}\text { BS } \\
(n=40)\end{array}$ & - & $16.48 \pm 3.06^{\mathrm{c}}$ & $31.18 \pm 2.03^{\mathrm{a}}$ & $\begin{array}{l}51.55 \quad \pm \\
4.10^{\mathrm{d}}\end{array}$ & $\begin{array}{l}44.10 \\
4.30^{\mathrm{ab}}\end{array}$ & $\begin{array}{l}2.20 \quad \pm \\
0.50^{\mathrm{d}}\end{array}$ & $3.60 \pm 0.60^{b}$ & NS \\
\hline $\begin{array}{l}\text { AADF } \\
(n=36)\end{array}$ & - & $18.21 \pm 6.11^{\mathrm{a}}$ & $\begin{array}{l}25.41 \pm \\
3.12^{\mathrm{d}}\end{array}$ & $\begin{array}{l}61.87 \\
5.10^{\mathrm{a}}\end{array}$ & $\begin{array}{l}38.30 \\
6.20^{\mathrm{c}}\end{array}$ & $\begin{array}{l}6.18 \quad \pm \\
0.40^{\mathrm{a}}\end{array}$ & $4.20 \pm 1.10^{\mathrm{a}}$ & NS \\
\hline $\mathbf{A T}$ & $\mathrm{CG}(\mathrm{n}=16)$ & $\begin{array}{c}17.87 \pm \\
4.23^{\mathrm{b}}\end{array}$ & $\begin{array}{c}25.56 \pm \\
3.11^{\mathrm{d}}\end{array}$ & $\begin{array}{ll}56.05 & \pm \\
5.10^{c} & \end{array}$ & $\begin{array}{l}38.70 \\
6.20^{c}\end{array}$ & $\begin{array}{l}6.36 \quad \pm \\
0.44^{\mathrm{a}}\end{array}$ & $4.14 \pm 1.02^{\mathrm{a}}$ & NS \\
\hline Day & $\mathrm{SG}(\mathrm{n}=16)$ & $\begin{array}{c}16.15^{ \pm} \\
3.22^{\mathrm{c}}\end{array}$ & $\begin{array}{c}28.51^{ \pm} \\
3.24^{\mathrm{c}}\end{array}$ & $\begin{array}{ll}56.06 & \pm \\
3.20^{c} & \end{array}$ & $\begin{array}{l}36.30 \\
5.40^{\mathrm{d}}\end{array}$ & $\begin{array}{l}5.18 \\
0.32^{\mathrm{b}}\end{array}$ & $3.90 \pm 1.04^{\mathrm{ab}}$ & NS \\
\hline AT & $\mathrm{CG}(\mathrm{n}=12)$ & $16.37 \pm 2.11^{\mathrm{c}}$ & $\begin{array}{c}27.12^{ \pm} \\
2.23^{\mathrm{d}}\end{array}$ & 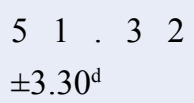 & $\begin{array}{l}43.20 \\
3.10^{\mathrm{b}}\end{array}$ & $\begin{array}{l}5.27 \quad \pm \\
0.40^{\mathrm{b}}\end{array}$ & $4.10 \pm 0.60^{\mathrm{ab}}$ & NS \\
\hline Day & $\mathrm{SG}(\mathrm{n}=12)$ & $\begin{array}{c}15.32 \pm \\
2.08^{\mathrm{d}}\end{array}$ & $\begin{array}{c}27.70 \pm \\
2.03^{\mathrm{cd}}\end{array}$ & $\begin{array}{l}45.04 \\
3.12^{\mathrm{f}}\end{array}$ & $\begin{array}{l}47.10 \\
3.40^{\mathrm{a}}\end{array}$ & $\begin{array}{l}4.04 \\
0.36^{c}\end{array}$ & $3.68 \pm 0.40^{b}$ & NS \\
\hline AT & $\mathrm{CG}(\mathrm{n}=8)$ & $\begin{array}{c}16.21^{ \pm} \\
1.24^{\mathrm{c}}\end{array}$ & $\begin{array}{c}28.04 \pm \\
1.16^{\mathrm{c}}\end{array}$ & $\begin{array}{l}53.16 \\
1.30^{\mathrm{de}}\end{array}$ & $\begin{array}{l}45.40 \\
2.16^{\mathrm{ab}}\end{array}$ & $\begin{array}{l}4.17 \\
0.23^{c}\end{array}$ & $4.06 \pm 0.34^{\mathrm{ab}}$ & NS \\
\hline Day & $\mathrm{SG}(\mathrm{n}=8)$ & $\begin{array}{c}14.25 \pm \\
1.00^{\mathrm{e}}\end{array}$ & $\begin{array}{c}28.14 \pm \\
0.68^{\mathrm{c}}\end{array}$ & $\begin{array}{l}50.13 \\
1.34^{\mathrm{d}}\end{array}$ & $\begin{array}{l}45.35 \\
2.10^{\mathrm{ab}}\end{array}$ & $\begin{array}{l}2.76 \\
0.10^{\mathrm{d}}\end{array}$ & $3.05 \pm 0.30^{\mathrm{c}}$ & NS \\
\hline AT & $\mathrm{CG}(\mathrm{n}=4)$ & $\begin{array}{c}15.60 \pm \\
0.55^{\mathrm{d}}\end{array}$ & $\begin{array}{c}27.43 \pm \\
0.55^{\mathrm{cd}}\end{array}$ & $\begin{array}{l}51.27 \\
0.57^{\mathrm{d}}\end{array}$ & $\begin{array}{l}43.38 \\
1.40^{\mathrm{b}}\end{array}$ & $\begin{array}{l}4.03 \\
0.20^{c}\end{array}$ & $4.00 \pm 0.30^{\mathrm{ab}}$ & NS \\
\hline Day & $\mathrm{SG}(\mathrm{n}=4)$ & $\begin{array}{c}16.21^{ \pm} \\
0.43^{\mathrm{c}}\end{array}$ & $\begin{array}{c}30.04 \pm \\
0.38^{\mathrm{b}}\end{array}$ & $\begin{array}{l}48.88 \\
0.42^{\mathrm{e}}\end{array}$ & $\begin{array}{l}46.30 \\
1.30^{\mathrm{a}}\end{array}$ & $\begin{array}{l}2.42 \quad \pm \\
0.10^{\mathrm{d}}\end{array}$ & $2.81 \pm 0.20^{\mathrm{d}}$ & NS \\
\hline
\end{tabular}

a-f: Different letters in the same column are statistically significant $(\mathrm{p}<0.05)$. BS: Before study, AADF: After atopic dermatitis formation, AT: After treatment, CG: Control group, SG: Study group, NS: Non-significant

Examining this Table, WBC, N, M, E and MCV levels showed a statistically significant increase ( $p$ $<0.05)$ after $\mathrm{AD}$, whereas a significant decrease $(\mathrm{p}$ $<0.05$ ) in RBC, HG, HCT, L, MCH and MCHC levels was observed. Although the mean of WBC, $\mathrm{N}, \mathrm{M}, \mathrm{E}$ and MCV showed a significant decrease ( $\mathrm{p}$ $<0.05$ ) in the comparisons in terms of time period, the mean of these parameters was lower in SG at all measurement times $(p<0.05)$ and the lowest levels were obtained on the last week of the study period, that is, on the 21 st day $(\mathrm{p}<0.05)$.

\section{Blood Biochemical Findings}

The average values of blood biochemical parameters measured in the current study are shown in Table 2. 


\begin{tabular}{|c|c|c|c|c|c|c|c|c|c|c|c|}
\hline 可 & $\begin{array}{l}\text { 命 } \\
\text { 茂 }\end{array}$ & 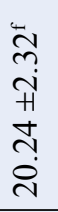 & 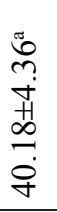 & 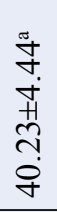 & $\begin{array}{l}\stackrel{0}{n} \\
\dot{+} \\
+ \\
0 \\
0 \\
\infty \\
m\end{array}$ & 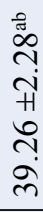 & 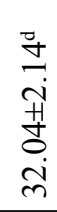 & 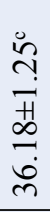 & 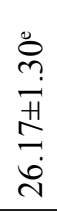 & 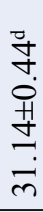 & 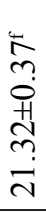 \\
\hline 겅 & $\begin{array}{l}\text { नि } \\
\text { स }\end{array}$ & 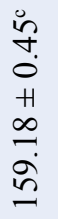 & 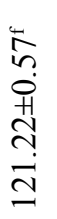 & $\begin{array}{l}0 \\
0 \\
n \\
0 \\
+1 \\
= \\
n \\
n \\
n\end{array}$ & 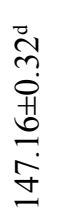 & 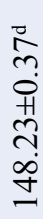 & $\begin{array}{l}\stackrel{0}{0} \\
\text { on } \\
\text { H } \\
\text { n. } \\
\infty \\
n \\
n\end{array}$ & 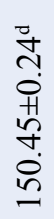 & 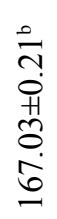 & 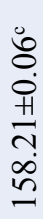 & 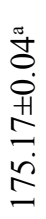 \\
\hline ₹ & $\begin{array}{l}\text { क् } \\
\text { स }\end{array}$ & 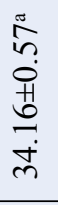 & 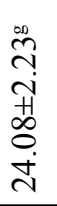 & 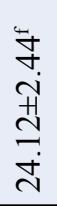 & 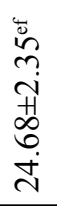 & 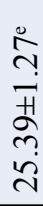 & 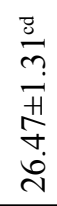 & 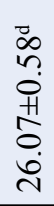 & 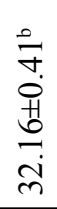 & 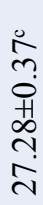 & 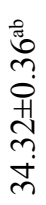 \\
\hline$\hat{\theta} \frac{\widehat{\theta}}{00}$ & $\begin{array}{l}\text { की } \\
\text { त } \\
x\end{array}$ & 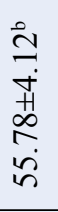 & 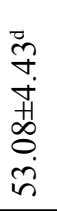 & $\begin{array}{l}\tilde{n} \\
\sim \\
+ \\
+ \\
\infty \\
\sim \\
\tilde{n} \\
n\end{array}$ & 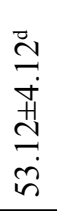 & 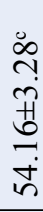 & 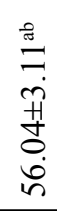 & $\begin{array}{l}\vec{D} \\
\hat{0} \\
\text { i } \\
\forall \\
\pm \\
\dot{0} \\
n\end{array}$ & 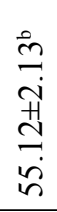 & 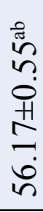 & 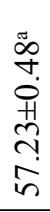 \\
\hline ย & $\begin{array}{l}\text { 命 } \\
\text { 艾 }\end{array}$ & \begin{tabular}{l} 
in \\
$n$ \\
$o$ \\
+1 \\
m \\
\multirow{r}{*}{}
\end{tabular} & 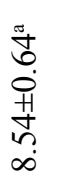 & $\begin{array}{l}\stackrel{\widetilde{\sigma}}{+} \\
0 \\
+ \\
\stackrel{+}{+} \\
\dot{\infty}\end{array}$ & 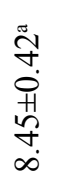 & $\begin{array}{l}\text { in } \\
\text { on } \\
\text { H } \\
\text { rn } \\
\stackrel{n}{r}\end{array}$ & 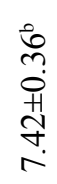 & 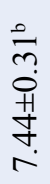 & 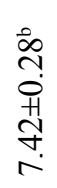 & $\begin{array}{l}\stackrel{ }{\Delta} \\
\frac{0}{0} \\
\frac{1}{\nabla} \\
r\end{array}$ & 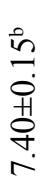 \\
\hline 빌 & $\begin{array}{l}\text { के } \\
\text { त } \\
\text { त }\end{array}$ & $\begin{array}{l}\stackrel{0}{I} \\
\vec{H} \\
\dot{\vec{b}} \\
\dot{r}\end{array}$ & 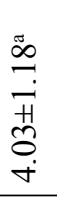 & $\begin{array}{l}\stackrel{\Xi}{\Xi} \\
\stackrel{+}{H} \\
\dot{\Delta} \\
\stackrel{+}{+}\end{array}$ & 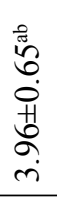 & 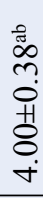 & 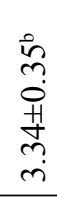 & 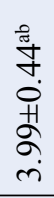 & $\begin{array}{l}\tilde{n} \\
\tilde{o} \\
\ddot{H} \\
\dot{y} \\
\dot{r}\end{array}$ & $\begin{array}{l}\infty \\
\frac{\infty}{0} \\
\stackrel{+}{+1} \\
\dot{\infty} \\
\dot{r}\end{array}$ & $\begin{array}{l}\vec{m} \\
\overrightarrow{0} \\
\dot{H} \\
\bar{\sigma} \\
\dot{i}\end{array}$ \\
\hline 商 & $\begin{array}{l}\text { की } \\
\text { त } \\
x\end{array}$ & 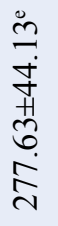 & 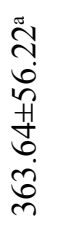 & 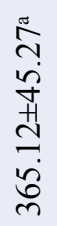 & 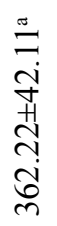 & 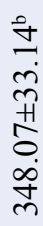 & 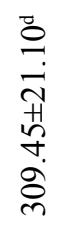 & 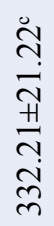 & 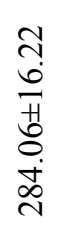 & 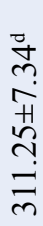 & 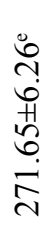 \\
\hline 光 & $\begin{array}{l}\text { की } \\
\text { त }\end{array}$ & 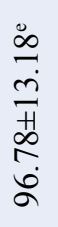 & $\begin{array}{l}\stackrel{\infty}{0} \\
\ddot{n} \\
\stackrel{+}{n} \\
\\
\ddot{\Xi} \\
=\end{array}$ & 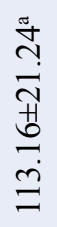 & 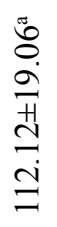 & $\begin{array}{l}\text { ते } \\
\text { in } \\
\text { Hु } \\
\stackrel{1}{1} \\
\infty \\
0 \\
0\end{array}$ & 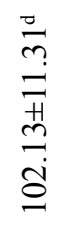 & 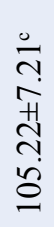 & 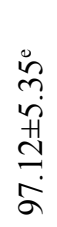 & 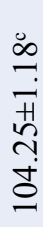 & $\begin{array}{l}\stackrel{\sim}{\sim} \\
\frac{1}{4} \\
\infty \\
\stackrel{2}{\infty}\end{array}$ \\
\hline 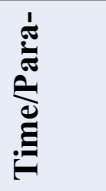 & 产 & ' & ' & 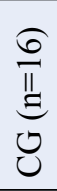 & 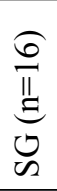 & $\underbrace{\stackrel{\overbrace{}}{J}}_{0}$ & 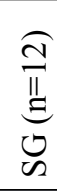 & $\underbrace{\stackrel{\infty}{\Perp}}_{0}$ & $\begin{array}{l}\underset{\infty}{\infty} \\
ٍ \\
ٍ \\
ٍ\end{array}$ & $\underbrace{\stackrel{\overbrace{}}{I}}_{0}$ & $\begin{array}{l}\underset{f}{\|} \\
\underset{\Xi}{J} \\
\text { J }\end{array}$ \\
\hline 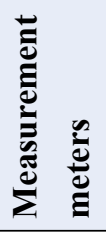 & & $\begin{array}{l}\underset{f}{+} \\
\stackrel{\Xi}{\Xi} \\
\infty\end{array}$ & $\underbrace{\substack{0 \\
\pi}}_{\frac{1}{2}}$ & & & & & & & & \\
\hline
\end{tabular}


When Table 2 is examined it was found that AST, LDH, GGT, OCT and IgE levels increased significantly $(\mathrm{p}<0.05)$ after $\mathrm{AD}$ formation, while TP, ALB and GLU levels decreased significantly $(p<0.05)$. With the start of the treatment period, it was observed that the levels of TP, ALB and GLU increased significantly in both groups $(\mathrm{p}<0.05)$, whereas AST, LDH, GGT, OCT and IgE levels decreased. However, the most important changes in increasing and decreasing parameters were found in SG mice on the 21st day of the study.

\section{Histopathological Findings}

In our study, necrosis in the epithelial structure and severe inflammation progressing up to lamina muscularis were detected after AD formation. Superficial perivascular infiltrate with lymphocytes, histiocytes and occasional neutrophils and eosinophils were also detected. In the comparisons at the end of the 21 st day, necrosis foci were encountered with severe inflammation and marked infiltration with $\mathrm{L}, \mathrm{N}$ and $\mathrm{E}$ along with tissue distortions formed due to $\mathrm{AD}$, were still continuing in $\mathrm{CG}$ group animals who were treated with tap water (Figure 1), whereas AD phenomenon had improved, inflamation and tissue distortion findings were resolved and epithelial tissue was normal in the Study Group animals who were treated with hot spring water.

\section{DISCUSSION AND CONCLUSION}

Atopic dermatitis is a chronic recurrent allergic inflammatory skin disease that affects millions of people worldwide, especially $10-20 \%$ of children worldwide and 1-3\% of adults (Bajgai et al., 2017). As an alternative, it has been reported that the treatment of AD with balneotherapeutic methods gives very successful results (Adler-Cohen et al., 2012). As a matter of fact, studies have shown that hot spring waters containing sulfur, manganese, magnesium and bicarbonate provide very successful results in the treatment of $A D$ as in other skin diseases (Valitutti et al., 1990). As a matter of fact, Süreyya I hot spring water and mud we use in treatment is a very rich water in terms of magnesium, bicarbonate, calcium, chlorine and many minerals (Elitok and Caliskan, 2020; Kislalioglu, 2019). Bajgai et al. (2017) reported that they obtained very successful results in a case

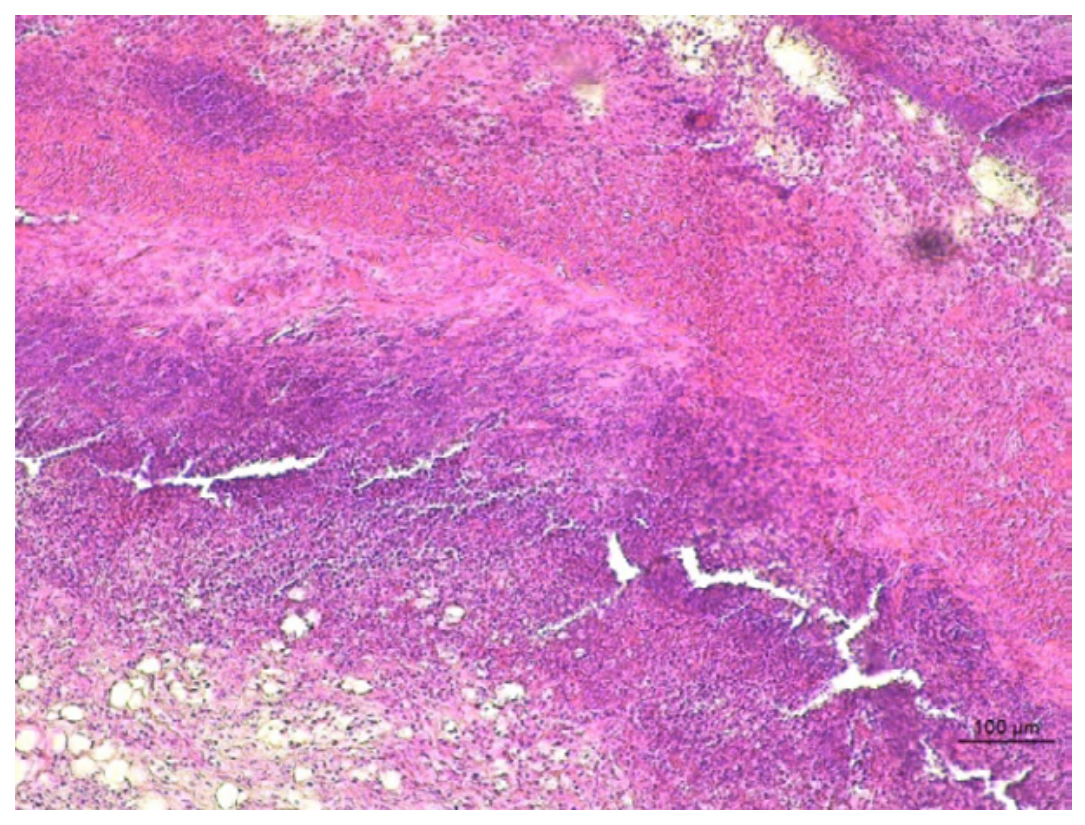

Figure 1 10x20 HE. Skin. Necrosis foci with severe inflammation (White arrows). (Control group, 21st day) 
similar to DNBC-induced AD in their study with hot spring water rich in minerals such as magnesium, calcium, chlorine and manganese. Inoue et al. (1999) reported that the balneotherapeutic effect of hot spring water was useful in controlling the clinical symptoms of acute exacerbations of $\mathrm{AD}$ cases. Similarly, we have determined the clinical therapeutic efficacy of Süreyya I hot spring water along with mud in DNBC-induced AD.

Kim et al. (2015) reported to have improved AD through the regulation of mineral water baths, Th2 production and the production of pro-inflammatory cytokines in DNCB-induced mice. Since IgE is a humoral reflection of Th2 immunity, Sureyya I hot spring water can be proposed as a reasonable mechanism of immunomodulation of spa spring water and sludge thanks to the data we obtained. Similarly, Bajgai et al. (2017) measured serum IgE levels to determine the immunological effects of their treatments with spa waters and found that $\operatorname{IgE}$ levels decreased over time in treated mice. Similar findings in terms of IgE levels were obtained in our study.

In this study, on the 21st day after treatment, it was observed that there was a statistically significant difference in terms of bw mean values $(p<0.05)$. This finding is in line with the findings obtained from a previous study (Lange et al., 2006), which claimed that treatment with hot spring water increased fat burning and caused fat loss in the intestine.

In the animals with $\mathrm{AD}$, body temperatures increased insignificantly ( $p>0.05)$, whereas the respiratory rate and cardiac frequency increased statistically significantly $(\mathrm{p}<0.05)$. These results were found similarly to findings previously made by Aghishi (1995).

In the study we conducted, statistically significant $(\mathrm{p}<0.05)$ differences were observed in the mean of WBC, L and $\mathrm{E}$ from hematological parameters after the application of DNBC. With the transition to the treatment period, it was determined that WBC, L and E levels gradually decreased in SG mice and the most important decrease occurred on the 21 st day. In cases of $\mathrm{AD}$, excessive infiltration of inflammatory cells and granular mast cells into the skin lesions, and high levels of IgE and WBC in the blood have been reported as characteristic hematological features of AD (Shirinbak et al., 2010). It had also been reported that hyperthermal waters $\left(<40{ }^{\circ} \mathrm{C}\right)$ showed an effect that suppressed the immune system, and the count of $\mathrm{T}$ lymphocytes in the blood decreased significantly in hyperthermal baths causing T-lymphocytopenia and eosinopenia by stimulating ACTH and cortisol production (Sukenik et al., 1997).

From the therapeutic point of view, $\mathrm{AD}$ is characterized by impaired skin barrier function, oxidative stress, insufficient immune system and increased IgE levels (Boguniewicz and Leung, 2010). In our study, higher than average $(p<0.05)$ mean of $\operatorname{IgE}$ titers obtained in all groups and times after the formation of $\mathrm{AD}$, support what these researchers reported. Again, from the same point of view, obtaining the lowest IgE levels in SG animals on the 21 st day of treatment can be interpreted as the most important improvement that took place in this group. This finding is accompanied by low E and WBC counts and histopathological findings.

In addition, it has been stated that $\mathrm{Mg}$ deficiency has pro-inflammatory effect and causes clinical inflammation syndrome resulting from leukocyte and macrophage activation and overproduction of free radicals (Pereira et al., 2014). Sureyya I hot spring water, which we use in the study, is rich in magnesium and suggests that magnesium may have an important role in the clinical recovery of chronic inflammation in animals and is accompanied by low WBC and L counts. 
In the present study, there were no significant differences between the groups in terms of $\mathrm{B}$. Actually, B is the least represented cell of the leukocyte order in all mammals (Siracusa et al., 2013). Accordingly, it is difficult to estimate basocytopenia value (Katica et al., 2020). There are no reports of $\mathrm{B}$ available in this context.

In our study, it was found that AST, LDH, GGT and OCT levels increased significantly $(\mathrm{p}<0.05)$, and TP, ALB and GLU levels decreased significantly $(p<0.05)$ after $\mathrm{AD}$ formation. With the treatment period beginning, it was observed that TP, ALB and GLU levels increased in CG and SG animals, whereas AST, LDH, GGT and OCT levels decreased. Similar findings have been previously declared by Cheon et al. (2013).

In our study, histopathological examinations of tissue sections taken from AD-created animals detected were necrosis and severe inflammatory foci that progressed to lamina muscularis.. On the 21 st day, it was seen that findings in the epidermis layer of the skin due to $\mathrm{AD}$ improved in $\mathrm{SG}$ animals treated with hot spring water and mud, whereas in CG mice treated with tap water, tissue deterioration along with the severe inflammation related to $\mathrm{AD}$

\section{REFERENCES}

Adler-Cohen C, Czarnowicki T, Dreiher J, Ruzicka T, Ingber A, Harari M, 2012. Climatotherapy at the Dead Sea: an effective treatment modality for atopic dermatitis with significant positive impact on quality of life. Dermatit, 23, 75-80.

Agishi Y, 1995. Hot springs and the physiological functions of humans. Asian Med J, 38, 115-24.

Bajgai J, Fadrıquela A, Ara J, Begum R, Ahmet MF, Kim CS, et al. 2017. Balneotherapeutic effects of high mineral spring water on the atopic dermatitis-like inflammation in hairless mice via immunomodulation and redox balance. BMC Complement Alltern Med, 17, 481-9. in epidermis still continued. Additionally, found were the necrosis foci. These findings have been found to be consistent with those reported by the researchers (Lee et al., 2016) who had proved that balneotherapy applications provided very successful results in the treatment of AD.

As a result from the point of clinical, hematological, blood biochemical and histopathological findings evaluated as a whole, the use of Süreyya I hot spring water along with mud has been shown to be very successful in the treatment of $\mathrm{AD}$.

\section{ACKNOWLEDEGMENTS}

The authors acknowledge to Mr. Suayp Demirel for his supports during the study, and Dr. Yavuz Ulusoy and Bahadir Kilic, Pathology Laboratory in R.T. Ministry of Agriculture and Forestry for histopathologic examinations.

\section{CONFLICT OF INTEREST}

None
Boguniewicz M, Leung DYM. 2010. Recent insights into atopic dermatitis and implications for management of infectious complications. J Allerg Clin Immunol, 125, 4-13.

Cheon C, Park S, Park JS, Oh SM, Jang S, Go HY, et al. 2013. KM110329 in adult patients with atopic dermatitis: a randomised, double-blind, placebo-controlled, multicentre trial-study protocol. BMC Complement Altern Med, 13, 33541.

Cozzi F, Lazzarin P, Todesco S, Cima L. 1995. Hypothalamic pituitary adrenal axis dysregulation in healthy subjects undergoing mud-bath application. Arthritis Rheum, 38, 72425.

Çalışkan D. 2019. Afyonkarahisar bölgesi kaplıca sularının farelerde deneysel oluşturulan diabetes mellitus üzerine 
tedavi edici etkilerinin araştırılması. Yüksek Lisans Tezi. Afyon Kocatepe Üniversitesi, Afyonkarahisar.

Elitok B. 2011. Kaplıcaların Mineral Mucizesi. Hancığlu Ofset, Afyonkarahisar.

Elitok B, Caliskan D. 2020. Investigation of therapeutic effects of hot spring waters sourced from Sureyya I (Turkey) on experimentally-induced diabetes mellitus in mice. Veterinaria, 69(3), 193-203.

Hwang JS, Kim JU, Yu YB, Im SH. 2013. Modulation of experimental atopic dermatitis bytopical application of GamiCheongyeul-Sodok-Eum. BMC Complement. Altern Med, 13, 312-22.

Inoune T, Inoune S, Kubata K. 1999. Bacterial activity of manganese and iodide ions against staphylococcus aureus: a possible treatment for acute atopic dermatitis. Acta Derm Venereol 79, 360-2.

Katica M, Janković O, Tandir F, Gradaščević N, Dekić R, Manojlović M, et al. 2020. The effects of calcium aluminate and calcium silicate cements implantation on haematological profile in rats. Kafkas Univ Vet Fak Derg, 26, 427-34.

Kim CG, Kang M, Lee YH, Min WG, Kim YH, Kang SJ, et al. 2015. Bathing effects of various seawaters on allergic (atopic) dermatitis-like skin lesions induced by2,4-dinitrochlorobenzene in hairless mice. Evid Based Complement Altern Med, 179185.

Kislalioglu I. 2019. Farelerde deneysel yolla oluşturulan karaciğer yağlanması üzerine afyon yöresi kaplıca sularının etkilerinin araştırılması. Yüksek Lisans Tezi. Afyon Kocatepe Üniversitesi, Afyonkarahisar.
Lange U, Muller-Ladner U, Schmidt KL. 2006. Balneotherapy in rheumatic diseases-an overview of novel and known aspects. Rheumatol Int, 26, 497-9.

Lee YB, Kim SJ, Park SM, Lee KH, Han HJ, Yu DS, et al. 2016. Immunomodulatory Effects of Deokgu Thermomineral Water Balneotherapy on Oxazolone-Induced Atopic Dermatitis Murine Model. Ann Dermatol, 28, 192-8.

Matz H, Orion E, Wolf R. 2003. Balneotherapy in dermatology. Dermatol Ther, 16, 132-40.

Pereira CD, Severo M, Araújo JR, Guımarães JT, Pestana D, Santos A, et al. 2014. Relevance of a hypersaline sodium-rich naturally sparkling mineral water to the protection against Metabolic Syndrome induction in fructose-fed SpragueDawley rats: a biochemical, metabolic and redox approach. Int J Endocrinol, 384583

Shirınbak S, Taher YA, Maazi H, Gras R, Van Esch BC, Henricks PA, et al. 2010. Suppression of Th2-driven airway inflammation by allergen immunotherapy is independent of B cell and Ig responses in mice. J Immunol, 185, 3857-65.

Siracusa MC, Kim BS, Spergel JM, Artis D. 2013. Basophils and allergic inflammation. J Allergy Clin Immunol, 132, 78988.

Suckow MA, Stevens KA. Wilso RP. 2012. The laboratory rabbit, guinea pig, hamster and other rodents. 1st ed. London, UK: Elsevier Inc.

Sukenik S, Abu-Shakra M, Flusser D. 1997. Balneotherapy in autoimmune diseases. Isr J Med Sci, 33, 258-61.

Valitutti S, Costellino F, Musiani P. 1990. Effect of sulphurous "thermal water" on Tlymphocytes proliferative response. Ann

Allergy, 65, 463-8. 


\section{ISPITIVANJE TERAPIJSKOG DJELOVANJA VODE IZ TERMALNIH IZVORA I BLATA NA EKSPERIMENTALNO IZAZVAN ATOPIJSKI DERMATITIS MIŠEVA}

\section{SAŽETAK}

Ovo istraživanje je provedeno sa ciljem procjene terapijskog djelovanja vruće izvorske vode i blata na atopijski dermatitis. U tu svrhu je korišteno 40 albino miševa iste životne dobi. Svi miševi su tretirani sa 2\% 2,4-dinitroklorbenzenom nakon deepilacije dorzalne regije u svrhu izazivanja atopijskog dermatitisa. Nakon izazivanja bolesti, započeo je period liječenja od 21. dan, pri čemu su miševi podjednako randomizirani u dvije grupe, kontrolnu i ispitivanu. Vršene su usporedbe između i unutar grupa kliničkih, hematoloških i biohemijskih krvnih parametara određenih prije i poslije nastanka atopijskog dermatitisa, i to 1, 7, 14. i 21. dana liječenja. Posljednjeg dana istraživanja, usporedbom sa kontrolnom grupom je uočen porast tjelesne težine $u$ ispitivanoj grupi zajedno sa signifikantnim $(\mathrm{p}<0.05)$ padom ukupnog broja leukocita, neutrofila, monocita, eozinofila, AST, LDH, GGT i IgE titra. Istovremeno je uočen izrazit porast $(p<0.05)$ srednjih vrijednosti srčane frekvence i frekvence disanja, broja eritrocita i limfocita, kao i hemoglobina i hematokrita. Nisu uočene signifikantne razlike između grupa u smislu broja bazofila. Posljednjeg dana uzorkovanja tkiva uočeno je da se oštećenje kože znantno poboljšalo $u$ ispitivanoj grupi životinja, dok je u kontrolnoj grupi miševa ono nastavljeno. Zaključeno je da se atopijski dermatitis poboljšao kod miševa koji su tretirani sa vrućom izvorskom vodom i blatom iz Süreyya.

Ključne riječi: Afyonkarahisar, atopijski dermatitis, balneoterapija, miš, terapija 